\title{
In Situ Two-Thermocouple Sensor Characterisation using Cross-Relation Blind Deconvolution with Signal Conditioning for Improved Robustness
}

\author{
Peter Hung ${ }^{1}$, Seán McLoone ${ }^{1}$, George Irwin ${ }^{2}$, Robert Kee ${ }^{2}$, and Colin Brown ${ }^{2}$ \\ ${ }^{1}$ Department of Electronic Engineering, National University of Ireland Maynooth, \\ Maynooth, Co. Kildare, Ireland \\ \{phung, sean.mcloone\}@eeng.nuim.ie \\ ${ }^{2}$ Virtual Engineering Centre, Queen's University Belfast, Belfast, BT9 5HN, \\ Northern Ireland \\ \{g.irwin, r.kee, cbrown17\}@qub.ac.uk
}

\begin{abstract}
Thermocouples are one of the most widely used temperature measurement devices due to their low cost, ease of manufacture and robustness. However, their robustness is obtained at the expense of limited sensor bandwidth. Consequently, in many applications signal compensation techniques are needed to recover the true temperature from the attenuated measurements. This, is turn, necessitates in situ thermocouple characterisation. Recently the authors proposed a novel characterisation technique based on the cross-relation method of blind deconvolution applied to the output of two thermocouples simultaneously measuring the same temperature. This offers a number of advantages over competing methods including low estimation variance and no need for a priori knowledge of the time constant ratio. A weakness of the proposed method is that it yields biased estimates in the presence of measurement noise. In this paper we propose the inclusion of a signal conditioning step in the characterisation algorithm to improve the robustness to noise. The enhanced performance of the resulting algorithm is demonstrated using both simulated and experimental data.
\end{abstract}

Keywords: Sensor, system identification, thermocouple, blind deconvolution

\section{Introduction}

In order to achieve high quality, low cost production with low environmental impact, modern industry is turning more and more to extensive sensing of processes and machinery, both for diagnostic purposes and as inputs to advanced control systems. Of particular interest in many applications is the accurate measurement of temperature transients in gas or liquid flows. For example, in an internal combustion engine the dynamics of the exhaust gas temperature is a key indicator of its performance as well as a valuable analytical input for on-board diagnosis of catalyst malfunction, while in the pharmaceutical industry precise control of transient temperatures is sometimes necessary in lypholisers used in drug manufacture to ensure the quality and consistency of the final product. These and many other applications, thus require the availability of fast response temperature sensors. 
Fast response temperature measurement can be performed using techniques such as Coherent Anti-Stokes Spectroscopy, Laser-Induced Fluorescence and Infrared Pyrometry [1] [2]. However, these are expensive, difficult to calibrate and maintain and are therefore impractical for wide-scale deployment outside the laboratory [2].

Thermocouples are widely used for temperature measurement due to their high permissible working limit and good linear temperature dependence. In addition, their low cost, robustness, ease of installation and reliability means that there are many situations in which thermocouples are indeed the only suitable choice. Unfortunately, their design involves a compromise between robustness and speed of response which poses major problems when measuring temperature fluctuations with high frequency signal components.

To remove the effect of the sensor on the measured quantity in such conditions, compensation of the thermocouple measurement is desirable. Usually, this compensation involves two stages: thermocouple characterisation followed by temperature reconstruction. Reconstruction is a process of restoring the unknown gas or fluid temperature from thermocouple outputs using either software techniques or hardware. This paper focuses on the first stage, since effective and reliable characterisation is essential for achieving satisfactory temperature reconstruction.

In an attempt to improve on existing thermocouple characterisation methods, the authors recently proposed a novel characterisation technique based on the crossrelation (CR) method of blind deconvolution [3] applied to the output of two thermocouples simultaneously measuring the same temperature [3] [4]. This offers a number of advantages over competing methods [2] [5] [6] [7] including low estimation variance and no need for a priori knowledge of the time constant ratio. However, a weakness of the proposed CR method is that it yields biased estimates in the presence of measurement noise [8]. This contrasts with its leading competitor, the Generalised Total Least Squares (GTLS) based difference equation characterisation algorithm [2] [9], which is an unbiased estimator but suffers from high estimation variance.

In this paper we propose a modification of the CR method that involves the inclusion of a signal conditioning step prior to the application of the CR algorithm, leading to improved robustness to measurement noise. The algorithm is validated using Monte Carlo simulations and data from an experimental test rig [10].

The remainder of the paper is organised as follows. The two-thermocouple characterisation methodology and the GTLS difference equation algorithm are introduced in Section 2. Section 3 provides an overview of the CR characterisation method and its principal characteristics. In Section 4 the CR implementation that incorporates signal conditioning filters is developed. The performance of this new algorithm is compared with the conventional CR implementation and the GTLS algorithm for both simulated and experimental data in Section 5. Finally, conclusions are presented in Section 6. 


\section{Difference Equation Sensor Characterisation}

\subsection{Thermocouple Modelling}

Provided a number of criteria regarding thermocouple construction are satisfied [5] [6], a first-order lag model with time constant $\tau$ and unity gain can represent the frequency response of a fine-wire thermocouple [11]. This simplified model can be written mathematically as

$$
T_{\mathrm{f}}(t)=T(t)+\tau \dot{T}(t)
$$

Here the original liquid or gas flow temperature $T_{\mathrm{f}}$ can be reconstructed if $\tau$, the thermocouple output $T(t)$ and its derivative are available. In practice, this direct approach is infeasible as $T(t)$ contains noise and its derivative is difficult to estimate accurately. More importantly, it is generally not possible to obtain a reliable a priori estimate of $\tau$, related to their thermocouple bandwidth $\omega_{B}$

$$
\tau=\frac{1}{\omega_{B}},
$$

which, in turn, is a function of thermocouple wire diameter $d$ and fluid velocity $v$

$$
\omega_{B} \propto \sqrt{\frac{v}{d^{3}}} .
$$

Hence, $\tau$ varies as a function of operating conditions. Clearly, a single thermocouple does not provide sufficient information for in situ estimation.

Equation (3) highlights the fundamental trade-off that exists when using thermocouples. Large wire diameters are usually employed to withstand harsh environments such as engine combustion systems, but these result in thermocouples with low bandwidth, typically $\omega_{B}<1 \mathrm{~Hz}$. In these situations high frequency temperature transients are lost with the thermocouple output significantly attenuated and phase-shifted compared to $T_{\mathrm{f}}$. Consequently, appropriate compensation of the thermocouple measurement is needed to restore the high frequency fluctuations.

\subsection{Two-Thermocouple Sensor Characterisation}

In 1936 Pfriem [12] suggested using two thermocouples with different time constants to obtain in situ sensor characterisation. Since then, various thermocouple compensation techniques incorporating this idea have been proposed in an attempt to achieve accurate and robust temperature compensation [2] [5] [6] [7] [13]. However, the performance of all these algorithms deteriorates rapidly with increasing noise power, and many are susceptible to singularities and sensitive to offsets [14].

Some of these two-thermocouple methods rely on the restrictive assumption that the ratio of the thermocouple time constants $\alpha(\alpha<1$ by definition) is known a priori. Hung et al. [2] [13] developed difference equation methods that do not require any $a$ 
priori assumption about the time constant ratio. The equivalent discrete time representation for the thermocouple model (1) is:

$$
T(k)=a T(k-1)+b T_{\mathrm{f}}(k-1),
$$

where $a$ and $b$ are difference equation ARX parameters and $k$ is the sample instant. Assuming ZOHs and sampling interval $\tau_{s}$, the parameters of the discrete and continuous time thermocouple models are related by

$$
a=\exp \left(-\tau_{s} / \tau\right), \quad b=1-a .
$$

For two thermocouples we have

$$
\begin{aligned}
& T_{1}(k)=a_{1} T_{1}(k-1)+b_{1} T_{\mathrm{f}}(k-1) \text { and } \\
& T_{2}(k)=a_{2} T_{2}(k-1)+b_{2} T_{\mathrm{f}}(k-1),
\end{aligned}
$$

where subscripts 1 and 2 are used to distinguish between signals from different thermocouples. The discrete time equivalent of the time constant ration $\alpha$ is then defined as

$$
\beta=b_{2} / b_{1}, \quad \beta<1 .
$$

The unknown temperature $T_{\mathrm{f}}$ can be eliminated from the thermocouple models (6) and (7) to give an expression in terms of $\beta, b_{2}$ and the thermocouple outputs only [2] [15], that is:

$$
\Delta T_{2}^{k}=\beta \Delta T_{1}^{k}+b_{2} \Delta T_{12}^{k-1},
$$

where the pseudo-sensor output $\Delta T_{2}^{k}$ and inputs $\Delta T_{1}^{k}$ and $\Delta T_{12}^{k-1}$ are defined as

$$
\begin{aligned}
\Delta T_{1}^{k} & =T_{1}(k)-T_{1}(k-1) \\
\Delta T_{2}^{k} & =T_{2}(k)-T_{2}(k-1) \\
\Delta T_{12}^{k-1} & =T_{1}(k-1)-T_{2}(k-1) .
\end{aligned}
$$

For an $M$-sample data set (9) can be expressed in ARX vector form

$$
\mathbf{Y}=\mathbf{X} \boldsymbol{\theta}
$$

with $\mathbf{Y}=\Delta \mathbf{T}_{2}^{k}, \mathbf{X}=\left[\Delta \mathbf{T}_{1}^{k} \Delta \mathbf{T}_{12}^{k-1}\right]$, and $\boldsymbol{\theta}=\left[\beta b_{2}\right]^{T}$. Here $\Delta \mathbf{T}_{1}^{k}, \Delta \mathbf{T}_{2}^{k}$ and $\Delta \mathbf{T}_{12}^{k-1}$ are vectors containing $M-1$ samples of the corresponding composite signals $\Delta T_{1}^{k}, \Delta T_{2}^{k}$ and $\Delta T_{12}^{k-1}$.

This characterisation model, referred to as the $\beta$-formulation, can be identified using least squares techniques. Due to the form of the composite input and output signals, the noise terms in the $\mathbf{X}$ and $\mathbf{Y}$ data blocks are not independent with the result that conventional least squares and total least squares both generate biased parameter estimates even when the measurement noise on the thermocouples is independent. However, by formulating identification as a generalised total least squares (GTLS) 
problem, unbiased parameter estimates can be obtained. The resulting $\beta$-GTLS algorithm is more robust than other difference equation formulations [15] and provides superior performance to other two-thermocouple probe characterisation methods at low and medium noise levels [2].

Unfortunately, the variance of $\beta$-GTLS estimates grows rapidly with increasing noise level, particularly when compared with conventional least squares [2]. In addition, the approach occasionally returns unreasonable time constant estimates at high noise levels, as noted in [4], due to ill-conditioning [16] and the sensitivity of the relationship between time constants and discrete model parameters (5) in the vicinity of the singularity at $b=0$. The cross-relation blind deconvolution approach proposed in [3] [4] avoids these issues.

\section{Blind Sensor Characterisation}

One of the best known deterministic blind deconvolution approaches is the method of cross-relation (CR) proposed by Liu et al. [17]. Such techniques exploit the information provided by output measurements from multiple systems of known structure but unknown parameters, for the same input signal.

This new approach to characterisation of thermocouples is completely different from those in Section 2. As commutation is a fundamental assumption for the method of cross-relation, the thermocouple models are both assumed to be linear. This is reasonably realistic as long as the thermocouples concerned are used within welldefined temperature ranges. Nonetheless, linearisation can easily be carried out using either the data capture hardware or software, even if the thermocouple response is nonlinear. Further, the approach requires constant model parameters, therefore the fluid or gas flow velocity $v$ is assumed to be constant, such that the two thermocouple time constants $\tau_{1}$ and $\tau_{2}$ are time-invariant.

\subsection{Two-Thermocouple Sensor Characterisation}

By exploiting the commutative relationship between linear systems, a novel twothemocouple characterisation scheme can be obtained as follows. Since the fluid temperature $T_{\mathrm{f}}$ is unknown, the two thermocouple output signals $T_{1}$ and $T_{2}$ are passed through two different synthetic thermocouples as shown in Fig. 1. These are also modelled by (1) and can be expressed in first-order transfer function form as:

$$
\hat{H}_{1}(s)=\frac{1}{1+s \hat{\tau}_{1}}, \quad \hat{H}_{2}(s)=\frac{1}{1+s \hat{\tau}_{2}},
$$

where $\hat{H}$ is the estimate of the thermocouple transfer function $H$. The unknown thermocouple time constant parameters can then be estimated as $\hat{\tau}_{1}$ and $\hat{\tau}_{2}$ using the cross-relation method, illustrated in Fig. 1. Here the cross-relation error signal, $e=T_{12}(t)-T_{21}(t)$ is used to define a mean-square-error cost function 


$$
\begin{aligned}
J_{\mathrm{MSE}}\left(\hat{\tau}_{1}, \hat{\tau}_{2}\right) & =E\left[e^{2}\right] \\
& =E\left[\left(T_{12}(t)-T_{21}(t)\right)^{2}\right], \forall \hat{\tau}_{1}, \hat{\tau}_{2} .
\end{aligned}
$$

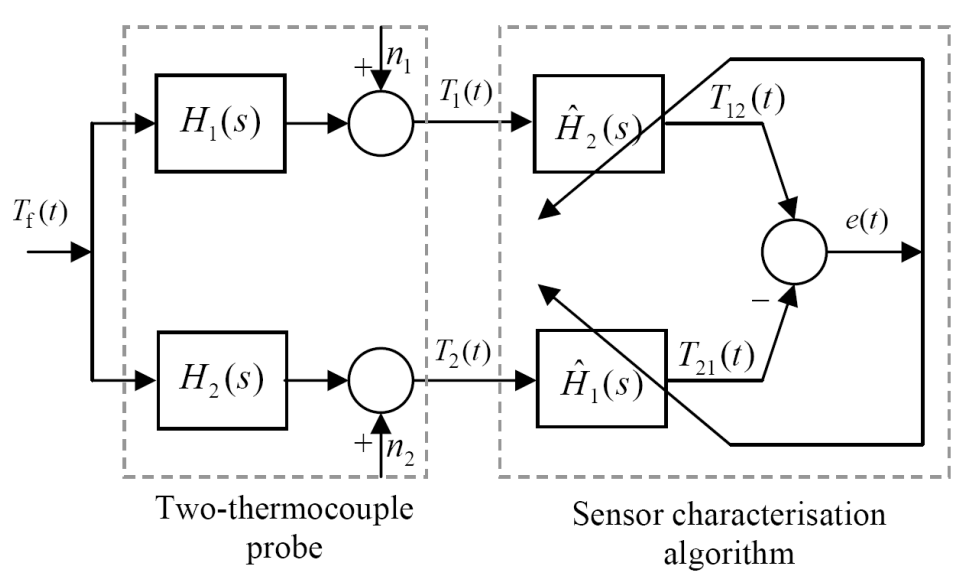

Fig. 1. Two-thermocouple cross-relation characterisation.

Equation (13) is then minimised with respect to $\hat{\tau}_{1}$ and $\hat{\tau}_{2}$ to yield the estimates of the unknown thermocouple time constants. Clearly, the cross-relation cost function $J_{\mathrm{MSE}}\left(\hat{\tau}_{1}, \hat{\tau}_{2}\right)$ is zero when $\hat{\tau}_{1}=\tau_{1}$ and $\hat{\tau}_{2}=\tau_{2}$. In practice it will not be possible to obtain an exact match between $T_{12}$ and $T_{21}$ due to measurement noise and other factors such as thermocouple modelling inaccuracy and violations of the assumption that the two thermocouples are experiencing identical environmental conditions.

$\mathrm{Xu}$ et al. [18] suggest that one of the necessary conditions for multiple finiteimpulse-response channels to be identifiable is that their transfer function polynomials do not share common roots. Applying this condition to the twothermocouple characterisation problem corresponds to requiring that the time constants, and hence the diameters (3), of the thermocouples are different, that is

$$
\tau_{1} \neq \tau_{2} \quad \Rightarrow \quad d_{1} \neq d_{2} .
$$

Not surprsingly, this requirement is consistent with all other two-thermocouple characterisation techniques mentioned in Section 2. Thus, cross-relation deconvolution converts the problem of sensor characterisation into an optimisation one.

\subsection{Cost Function}

A 3-D surface plot and a contour map of a typical $J_{\mathrm{MSE}}\left(\hat{\tau}_{1}, \hat{\tau}_{2}\right)$ cost function are shown in Fig. 2. Unfortunately, $J_{\mathrm{MSE}}\left(\hat{\tau}_{1}, \hat{\tau}_{2}\right)$ is not quadratic and cannot therefore be minimised using linear least squares. More importantly, the cost function has a second minimum when both time constant values approach infinity. Under these conditions, 
both low-pass filters (12) take infinite amounts of time to respond. In other words, they are effectively open-circuited and their differences will always be zero. The existence of this minimum applies regardless of the noise conditions or any violations of the modelling assumptions. The minimum at infinity is thus in fact the global minimum, while the true time constant value is located at a local minimum. In the absence of noise, it is noted that $J_{\mathrm{MSE}}=0$ at both the global and local minima.

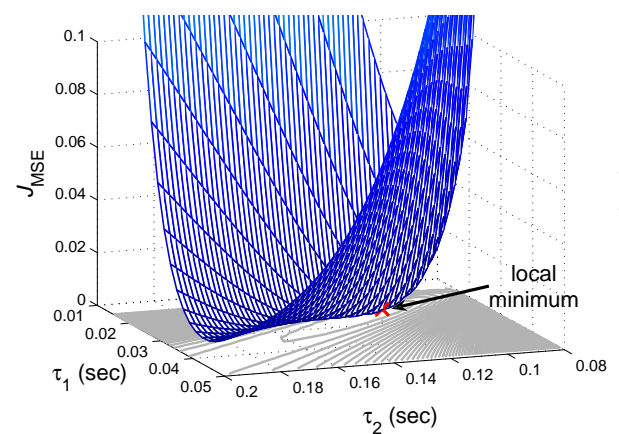

(a)

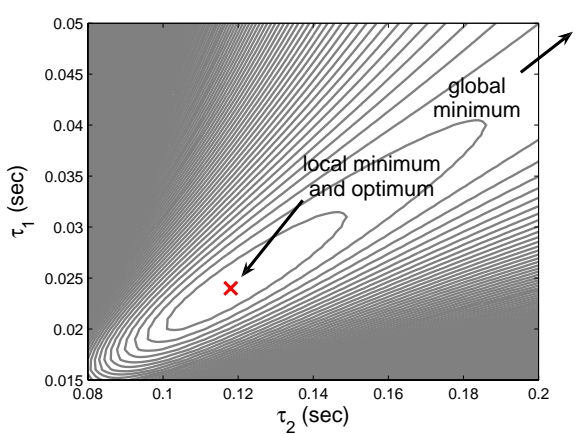

(b)

Fig. 2. A typical $J_{\mathrm{MSE}}$ cost function for noiseless thermocouple measurements: (a) 3-D plot of cost function; and (b) corresponding contour map.

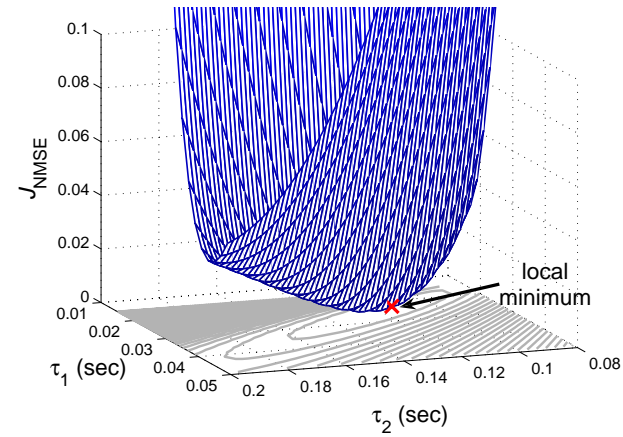

(a)

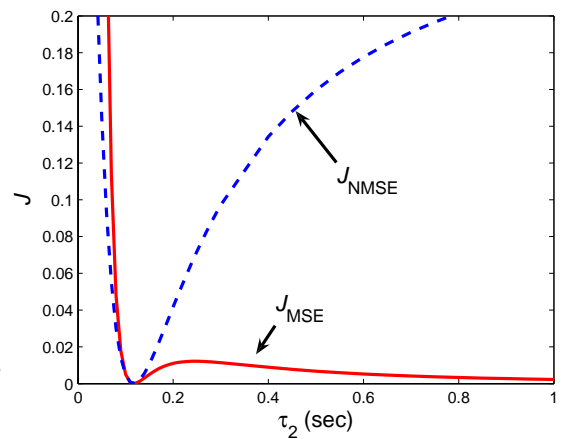

(b)

Fig. 3. A typical $J_{\mathrm{NMSE}}$ cost function for noiseless thermocouple measurements: (a) 3-D plot; and (b) a comparison of 1-D cross sections of the MSE and NMSE CR cost functions.

The narrow basin of attraction of the desired local minimum coupled with the global minimum at infinity has serious implications for optimisation complexity since search bounds have to be carefully selected to avoid divergence of gradient search algorithms to the global minimum. Further, with increasing noise level the local minima becomes shallower and shallower, and eventually disappears causing the optimisation problem to become ill-posed.

As noted in [3] the ill-posed problem can be resolved by employing a normalised mean squared error (NMSE) cost function defined as 


$$
J_{\mathrm{NMSE}}\left(\hat{\tau}_{1}, \hat{\tau}_{2}\right)=\frac{E\left[\left(T_{12}(t)-T_{21}(t)\right)^{2}\right]}{0.5\left[\operatorname{var}\left(T_{12}\right)+\operatorname{var}\left(T_{21}\right)\right]} .
$$

A typical example of this cost function is plotted in Fig. 3(a). To highlight the effect of normalisation, the 1-D cross sections of both the MSE and NMSE cost functions along the line $\hat{\tau}_{1}=\alpha \hat{\tau}_{2}$ is also plotted in Fig. 3(b). Essentially, normalisation penalises large time constants, thereby eliminating the minimum at infinity giving a well conditioned convex cost function.

A weakness of the MSE and NMSE cross-relation algorithms is that they generate biased estimates. In fact, a statistical analysis of the algorithms [8] reveals that the MSE implementation yields postiviely biased estimates, while the NMSE implementation results in negatively biased estimates at high noise levels, though the latter is less significant when temperature variation is broadband.

\section{$4 \quad$ Signal Conditioning}

One approach to reducing the noise induced estimation bias is to introduce signal conditioning filters $\left(F_{c}(s)\right)$ prior to the $\mathrm{CR}$ characterisation algorithm as illustrated in Fig. 4. Provided the filters are identical, linear (thereby ensuring commutativity) and do not completely block the measured signals, the operation of the $\mathrm{CR}$ algorithm is unaffected. Within these constraints there is substantial freedom in the design of the filters.

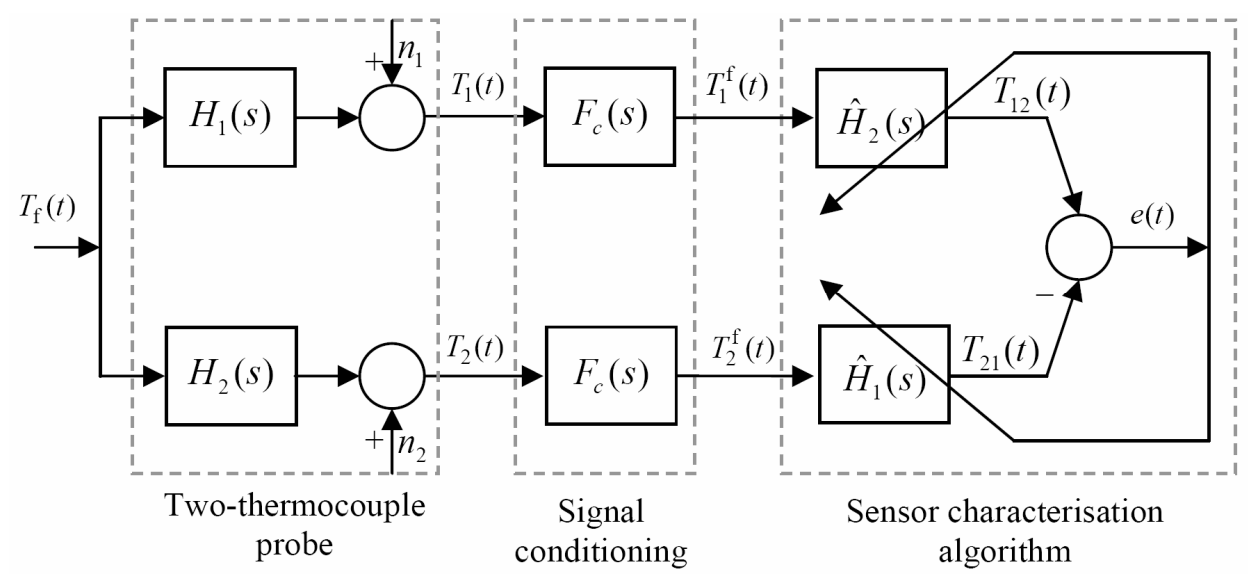

Fig. 4. Two-thermocouple cross-relation characterisation with signal conditioning

Assuming white measurement noise, which has a constant power spectrum profile across all frequencies, the obvious choice is to match the passband of the conditioning filters to the bandwidth of the temperature fluctuations. However, this is not the optimum choice, since it does not take into account the effect of the thermocouples. 
Consider the magnitude squared transfer function $|G(j \omega)|^{2}$ from the input signal, $T_{\mathrm{f}}$, to the cross-relation (CR) error signal, $e$, when $\hat{H}_{1}=\hat{H}_{2}=1$, defined as

$$
|G(j \omega)|^{2}=\left|\frac{T_{1}(j \omega)-T_{2}(j \omega)}{T_{\mathrm{f}}(j \omega)}\right|^{2}=\left|H_{1}(j w)-H_{2}(j w)\right|^{2} .
$$

This is plotted in Fig. 5 as a function frequency for a typical two-thermocouple sensor. As can be seen in Fig. 5, $|G(j \omega)|^{2}$ has a peak between the thermocouple cutoff frequencies (i.e. between $1 / \tau_{2}$ and $1 / \tau_{1}$ ) and decays rapidly towards zero away from this peak. On the right hand side the decay is due to the increasing attenuation of the thermocouple signals at higher frequencies. On the left hand side, however, the decay occurs because there is less and less difference between thermocouple signals while moving into the passband of the lowest bandwidth thermocouple (i.e. $\left.<1 / \tau_{2}\right)$.

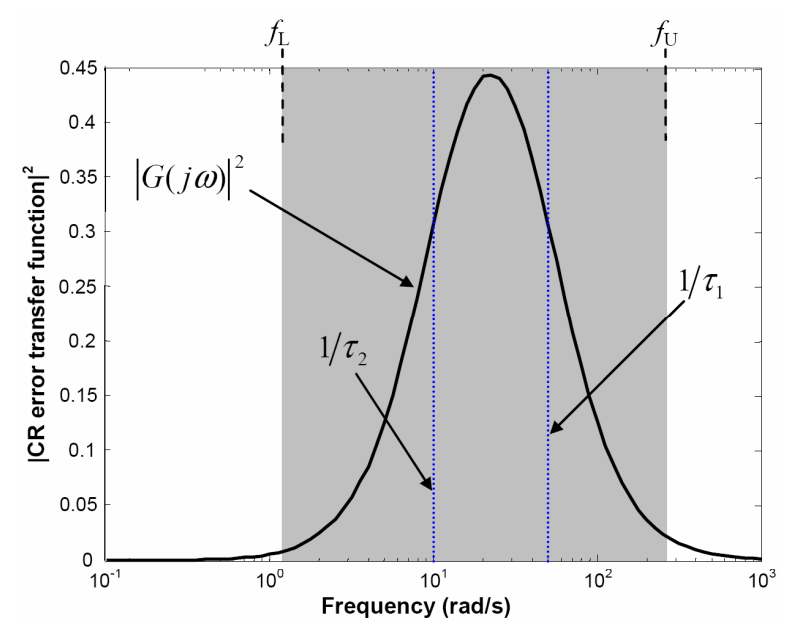

Fig. 5. Normalised Cross-relation error transfer function as a function of frequency for a twothermocouple probe with time constants 0.02 and 0.1 seconds respectively.

The dynamic range of the CR error transfer function is approximately $0.1 / \tau_{2}$ to $10 / \tau_{1} \mathrm{rad} / \mathrm{s}$. Thus, the effective CR error signal bandwidth will be limited to the intersection of the passband of $G(j \omega)$ and the input signal bandwidth, and as such will, in general, be substantially less than the signal bandwidth. Consequently, for optimum signal-to-noise ratio performance the signal conditioning filters should be band-pass filters with a lower cut-off frequency, $0<f_{\mathrm{L}} \leq \max \left(0.1 / \tau_{2}, f_{\min }\right)$ and an upper cut-off frequency, $1 / \tau_{1}<f_{\mathrm{U}} \leq \min \left(10 / \tau_{1}, f_{\max }\right)$. Here, $f_{\min }$ and $f_{\max }$ are the minimum and maximum frequencies of the temperature fluctuations $\left(T_{\mathrm{f}}\right)$. The maximum frequency $f_{\max }$ is assumed to be greater than the bandwidth of the faster thermocouple $\left(1 / \tau_{1}\right)$, otherwise signal compensation would not be required. In general, temperature fluctuations will be low-pass, in which case $f_{\min }=0$ and $f_{\max }$ corresponds to the signal bandwidth. 


\section{Performance Evaluation}

To evaluate the performance of the proposed signal conditioned CR algorithm (SCCR) against conventional CR characterisation (CR) and the GTLS difference equation approach ( $\beta$-GTLS), Monte Carlo simulations were performed using data from a two-thermocouple probe simulated in MATLAB ${ }^{\circledR}$ and an experimental test rig.

In the simulation the thermocouples were modelled as unity gain first-order lowpass filters with time constants $\tau_{1}=23.8$ and $\tau_{2}=116.8 \mathrm{~ms}$, respectively, and connected to a common input representing the fluctuating gas or liquid temperature signal. Data sets were generated for a sinusoidally varying temperature profile,

$$
T_{\mathrm{f}}(t)=16.5 \sin (20 \pi t)+50.5,
$$

and a band-limited white noise signal obained by low-pass filtering the output of MATLAB's normally distributed random signal generator using a $125 \mathrm{rad} / \mathrm{s}$ bandwidth second-order butterworth filter. Samples of each signal, along with the corresponding thermocouple measurements are given in Fig. 6. Each data set was recorded after initial condition transients had decayed and consisted of 5000 points at a sampling interval of $2 \mathrm{~ms}$.

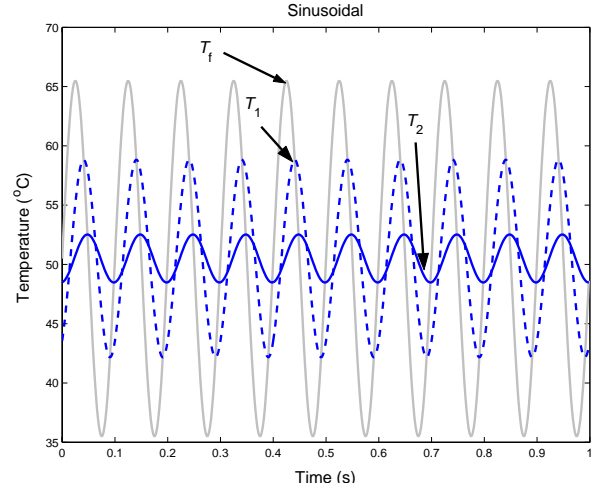

(a)

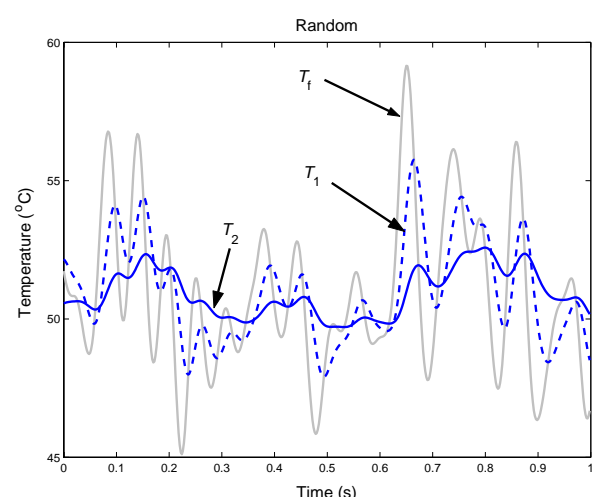

(b)

Fig. 6. Simulated temperature profiles: (a) sinusoidal; and (b) random band-limited to $20 \mathrm{~Hz}$.

The test rig, depicted in Fig. 7, was specifically designed to produce periodic temperature fluctuations at constant fluid velocity [10]. It is supplied with air through a pressure regulator and a needle valve in order to obtain approximately constant mass flow rate. The flow is divided into two streams, one heated and the other at the supplied temperature. The streams are balanced using ball valves to ensure a uniform velocity profile across the air outlet. Both streams are then passed to isolated reservoirs before leaving their corresponding orifices. Finally, the warm and cool streams are combined in the mixing chamber before reaching the temperature probe. The frequency of periodic temperature fluctuations is controlled by the frequency of crank rotation that is connected to the rig via a linkage. The temperature probe 
consists of two thermocouples of unequal diameters (50 and $127 \mu \mathrm{m})$ and a constantcurrent anemometer $(3.8 \mu \mathrm{m})$ used to provide a reference measurement.

Using this test rig data was collected for periodic temperature fluctuations with a fundamental frequency of $38 \mathrm{rad} / \mathrm{s}$ at a sampling frequency of $1 \mathrm{kHz}$ (Fig. 8(a)). Table 1 shows the time constant estimates obtained with each of the three characterisation methods. For comparison purposes, the best estimate of the time constants using the anemometer signal as an approximation to the true temperature is also included in the table. This essentially represents a lower bound on the true time constant values.

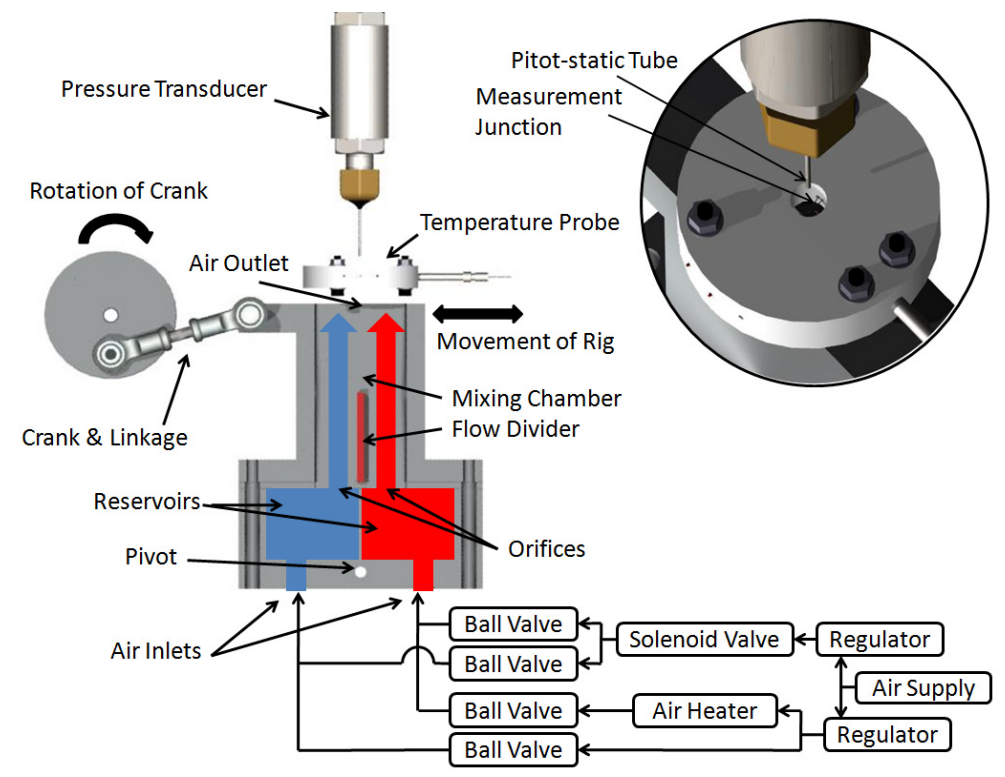

Fig. 7. Test rig schematic.

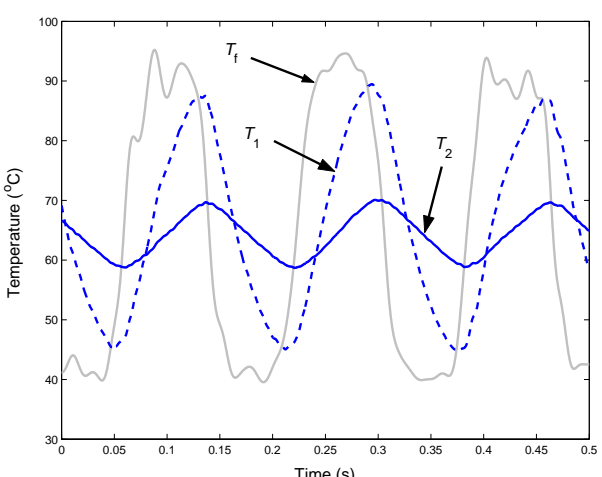

(a)

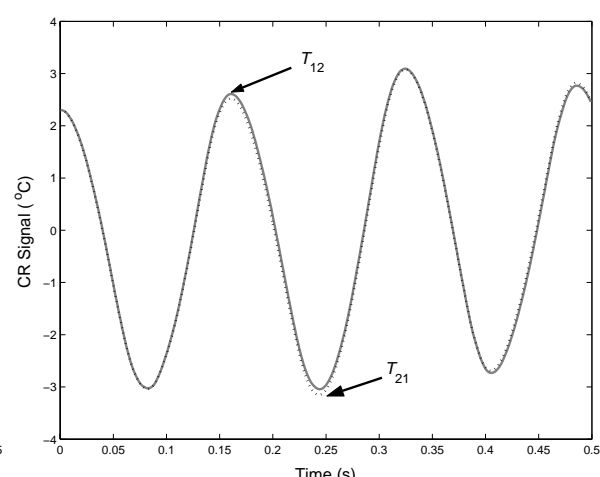

(b)

Fig. 8. Test rig data: (a) temperature profiles, and; (b) comparison of CR signals. 
Table 1. Time constant estimates for test rig data

\begin{tabular}{|c|r|r|r|r|}
\hline Method & Ref. fit & $\boldsymbol{\beta}$-GTLS & CR & SCCR \\
\hline$\tau_{1}$ estimate $(\mathrm{ms})$ & 37.9 & 37.4 & 38.0 & 37.7 \\
\hline$\tau_{2}$ estimate $(\mathrm{ms})$ & 187.9 & 185.3 & 187.4 & 185.8 \\
\hline
\end{tabular}

As can be seen, the results are consistent across all methods, suggesting that the data has low noise contamination and is consistent with the two-thermocouple probe modelling assumptions. The is further confirmed by the close fit obtained between the CR signals ( $T_{12}$ and $T_{21}$ from Fig. 1) as shown in Fig. 8(b). Consequently, for the purposes of the Monte Carlo simulations, the 500 point test rig data set is taken to be essentially noise free with $\tau_{1} \approx 38$ and $\tau_{2} \approx 188 \mathrm{~ms}$.

For each of the three data sets 100-run Monte Carlo simulations were performed for zero-mean white Gaussian measurement noise added to the noise free thermocouple outputs. The amount of noise added was quantified in terms of the noise level $L_{e}$, defined as

$$
L_{e}=\sqrt{\frac{\operatorname{var}\left(n_{i}\right)}{\operatorname{var}\left(T_{\mathrm{f}}\right)}} \cdot 100 \%, \quad i=1,2,
$$

where $n_{1}$ and $n_{2}$ are the noises added to the thermocouple measurements. For a given $L_{e}$, the performance of each characterisation algorithm was assessed in terms of the percentage error in estimating the time constants, that is:

$$
e_{\tau_{i}}=\frac{\hat{\tau}_{i}-\tau_{i}}{\tau_{i}} \cdot 100 \%, \quad i=1,2
$$

The means and standard deviations of this estimation error are recorded for a range of noise levels in Table 2 for each of the characterisation algorithms under consideration (SCCR, CR and $\beta$-GTLS). In SCCR the bandwidth of the conditioning filters was chosen as $f_{\mathrm{L}}=60, f_{\mathrm{U}}=90 \mathrm{rad} / \mathrm{s}$ for the sinusoidal data, $f_{\mathrm{L}}=5, f_{\mathrm{U}}=120 \mathrm{rad} / \mathrm{s}$ for the random data and $f_{\mathrm{L}}=25, f_{\mathrm{U}}=125 \mathrm{rad} / \mathrm{s}$ for the test rig data. Note that results for $\hat{\tau}_{2}$ have been omitted as they show a similar pattern to those observed for $\hat{\tau}_{1}$.

\section{Discussion and Conclusions}

The results clearly show that the inclusion of signal conditioning filters has the desired effect. SCCR consistently has much lower bias than CR, particularly at higher noise levels. The picture for variance is less clear. SCCR estimates have slightly greater variance on average than the $\mathrm{CR}$ estimates for the simulated data, but substantially less variance in the case of the test rig data. This is currently the subject of further study.

While $\beta$-GTLS is theoretically unbiased, the variance in the estimates grows very rapidly with noise, and the algorithm essentially breaks down for $L_{e}>5$ in the simulated examples and $L_{e}>1$ for the test rig data. The substantially worse $\beta$-GTLS 
results in the last problem are due to the higher sample rate and fewer data points in this problem, both of which amplify the sensitivity of GTLS to noise. In contrast, CR and SCCR perform very well on this problem, though the estimation variance is significantly higher than in the simulated examples due to the smaller number of data points (500 compared to 5000). In practice, pre-filtering of the data can substantially improve the robustness of GTLS to noise at the expense of introducing some bias [2], but this has not been investigated here due to space constraints.

The cross-relation (CR) method of blind deconvolution provides an attractive framework for two-thermocouple sensor characterisation. It does not require a priori knowledge of the thermocouple time constant ratio $\alpha$, as required in many other characterisation algorithms, though this information can be exploited if available. CR is more noise-tolerant in the sense of reduced parameter estimation variance when compared to the alternatives such as $\beta$-GTLS. The standard CR implementation yields biased estimates, but this is significantly reduced with the inclusion of signal conditioning filters. The resulting SCCR algorithm has been shown to be superior to other methods on both simulated and experimental data.

Table 2. Means and (standard deviations) of $\hat{\tau}_{1}$ estimation errors (\%) obtained with $\beta$-GTLS, $\mathrm{CR}$ and SCCR for each data set for a range of noise levels

\begin{tabular}{|c|c|c|c|c|c|c|c|}
\hline $\begin{array}{c}\text { Noise Level } \\
\left(\boldsymbol{L}_{\boldsymbol{e}}\right)\end{array}$ & $\mathbf{1}$ & $\mathbf{3}$ & $\mathbf{5}$ & $\mathbf{7}$ & $\mathbf{1 0}$ & $\mathbf{1 5}$ & $\mathbf{2 0}$ \\
\hline \multicolumn{8}{|c|}{ Sinusoidal simulation } \\
\hline \multirow{2}{*}{$\beta$-GTLS } & -0.17 & -0.77 & -0.57 & 3.14 & 5.47 & 35.85 & -6.43 \\
& $(0.69)$ & $(5.29)$ & $(13.90)$ & $(26.93)$ & $(60.83)$ & $(318.50)$ & $(969.42)$ \\
\hline \multirow{2}{*}{ CR } & -0.13 & -1.64 & -3.95 & -6.94 & -12.10 & -19.78 & -26.3 \\
& $(0.32)$ & $(1.01)$ & $(1.56)$ & $(2.39)$ & $(3.22)$ & $(4.29)$ & $(4.43)$ \\
\hline \multirow{2}{*}{ SCCR } & -0.07 & -0.36 & -0.57 & -1.53 & -2.57 & -5.68 & -9.53 \\
& $(0.36)$ & $(1.02)$ & $(1.58)$ & $(2.41)$ & $(3.25)$ & $(4.70)$ & $(6.43)$ \\
\hline \multicolumn{7}{|c|}{ Random simulation } \\
\hline \multirow{2}{*}{$\beta$-GTLS } & -0.01 & 0.33 & 1.27 & 5.23 & 17.12 & 49.57 & 98.31 \\
& $(0.96)$ & $(7.62)$ & $(20.27)$ & $(42.64)$ & $(88.72)$ & $(465.72)$ & $(549.96)$ \\
\hline \multirow{2}{*}{ CR } & 0.01 & -0.34 & 0.73 & 1.55 & 2.87 & 5.27 & 10.18 \\
& $(0.21)$ & $(0.71)$ & $(1.24)$ & $(1.37)$ & $(2.39)$ & $(3.36)$ & $(4.39)$ \\
\hline \multirow{2}{*}{ SCCR } & -0.04 & 0.22 & -0.08 & 0.53 & 1.44 & 2.51 & 5.09 \\
& $(0.33)$ & $(0.97)$ & $(1.54)$ & $(2.28)$ & $(3.07)$ & $(4.80)$ & $(6.60)$ \\
\hline \multicolumn{7}{|c|}{ Test rig } \\
\hline \multirow{2}{*}{$\boldsymbol{\beta}$-GTLS } & 0.05 & 20.84 & -127.97 & -180.31 & -281.23 & -229.94 & -168.53 \\
& $(9.76)$ & $(89.11)$ & $(1237.54)$ & $(1028.05)$ & $(697.22)$ & $(604.96)$ & $(1000.33)$ \\
\hline \multirow{2}{*}{ CR } & -1.72 & -1.08 & -1.21 & -1.83 & -4.43 & -3.35 & -2.51 \\
& $(1.29)$ & $(4.23)$ & $(6.12)$ & $(7.50)$ & $(11.50)$ & $(19.73)$ & $(31.30)$ \\
\hline \multirow{2}{*}{ SCCR } & -0.94 & -0.98 & -0.28 & -0.59 & -1.67 & -1.87 & 1.57 \\
& $(1.03)$ & $(2.89)$ & $(4.51)$ & $(5.90)$ & $(9.68)$ & $(14.59)$ & $(19.74)$ \\
\hline
\end{tabular}




\section{References}

1. Kee, R. J., Blair, G. P.: Acceleration test method for a high performance two-stroke racing engine. In: SAE Motorsports Conference, Detroit, MI, Paper No. 942478 (1994)

2. Hung, P. C., McLoone, S., Irwin G., Kee, R.: A difference equation approach to twothermocouple sensor characterisation in constant velocity flow environments. Rev. Sci. Instrum. 76, Paper No. 024902 (2005)

3. Hung, P. C., Kee, R. J., Irwin G. W., McLoone, S. F.: Blind Deconvolution for TwoThermocouple Sensor Characterisation. ASME Dyn. Sys. Measure. Cont. 129, 194--202 (2007)

4. Hung, P. C., McLoone, S. F., Irwin, G. W., Kee, R. J.: Blind Two-Thermocouple Sensor Characterisation. In: International Conference on Informatics Control, Automation and Robotics (ICINCO 2007), Angers, France, pp.10--16 (2007)

5. Forney, L. J., Fralick G. C.: Two wire thermocouple: Frequency response in constant flow. Rev. Sci. Instrum. 65, 3252--3257 (1994)

6. Tagawa, M., Ohta, Y.: Two-Thermocouple Probe for Fluctuating Temperature Measurement in Combustion - Rational Estimation of Mean and Fluctuating Time Constants. Combust. and Flame. 109, 549--560 (1997)

7. Kee, R. J, O'Reilly, P. G., Fleck, R., McEntee, P. T.: Measurement of Exhaust Gas Temperature in a High Performance Two-Stroke Engine. SAE Trans. J. Engines. 107, Paper No. 983072 (1999)

8. McLoone, S. F., Hung, P. C., Irwin, G. W., Kee, R. J.: On the stability and biasedness of the cross-relation blind thermocouple characterisation method. In: IFAC World Congress 2008, Seoul, South Korea, submitted (2008)

9. McLoone, S., Hung, P., Irwin, G., Kee, R.: Difference equation sensor characterisation algorithms for two-thermocouple probes. Trans. InstMC, accepted (2008)

10.Brown, C., Kee, R. J., Irwin, G. W., McLoone, S. F., Hung, P.: Identification Applied to Dual Sensor Transient Temperature Measurement. In: UKACC Control 2008, Manchester, UK, submitted (2008)

11.Petit, C., Gajan, P., Lecordier, J. C., Paranthoen, P.: Frequency response of fine wire thermocouple. J. Phy. Part E. 15, 760--764 (1982)

12.Pfriem, H.: Zue messung verandelisher temperaturen von ogasen und flussigkeiten. Forsch. Geb. Ingenieurwes. 7, 85--92 (1936)

13.Hung, P., McLoone, S., Irwin G., Kee, R.: A Total Least Squares Approach to Sensor Characterisations. In: 13th IFAC Symposium on Sys. Id., Rotterdam, The Netherlands, pp. 337--342 (2003)

14.Kee, J. K., Hung, P., Fleck, B., Irwin, G., Kenny, R., Gaynor, J., McLoone, S.: Fast response exhaust gas temperature measurement in IC Engines. In: SAE 2006 World Congress, Detroit, MI, Paper No. 2006-01-1319 (2006)

15.McLoone, S., Hung, P., Irwin, G., Kee, R.: Exploiting A Priori Time Constant Ratio Information in Difference Equation Two-Thermocouple Sensor Characterisation. IEEE Sensors J. 6, 1627--1637 (2006)

16.Van Huffel S., Vandewalle, J.: The Total Least Squares Problem: Computational Aspects and Analysis, SIAM, Philadelphia, 1st edition (1991)

17.Liu, H., Xu, G., Tong, L.: A deterministic approach to blind identification of multichannel FIR systems. In: 27th Asilomar Conference on Signals, Systems and Computers, Asilomar, CA, pp. 581--584 (1993)

18.Xu, G., Liu, H., Tong, L., Kailath, T.: A least-squares approach to blind channel identification. IEEE Trans. Signal Proc. 43, 2982--2993 (1995) 